\title{
A practical approach for quantitative estimates of voxel-by-voxel liver perfusion using DCE imaging and a compartmental model
}

\author{
Yue Cao \\ Departments of Radiation Oncology and Radiology, University of Michigan, Ann Arbor, Michigan 48109 \\ Jonathan Alspaugh, Zhou Shen, James M. Balter, \\ Theodore S. Lawrence, and Randall K. Ten Haken \\ Department of Radiation Oncology, University of Michigan, Ann Arbor, Michigan 48109
}

(Received 24 January 2006; revised 12 June 2006; accepted for publication 14 June 2006; published 28 July 2006)

\begin{abstract}
Voxel-by-voxel estimation of liver perfusion using nonlinear least-squares fits of dynamic contrast enhanced computed tomography or magnetic resonance imaging data to a compartmental model is a computational expensive process. In this report, a "linear" least-squares method for estimation of liver perfusion is described. Simulated data and the data from an example case of a patient with intrahepatic cancer are presented. Compared to the nonlinear method, the new method can improve computational speed by a factor of $\sim 400$, which makes it practical for use in clinical trials. () 2006 American Association of Physicists in Medicine. [DOI: 10.1118/1.2219773]
\end{abstract}

Key words: liver perfusion, a single compartmental model, CT perfusion, linear least squares

\section{INTRODUCTION}

Measurement of organ perfusion from dynamic contrast enhanced imaging (DCE), including positron emission tomography (PET), ${ }^{1}$ computed tomography $(\mathrm{CT}),{ }^{2-4}$ and magnetic resonance imaging (MRI) ${ }^{5,6}$ is an emerging methodology for diagnosis and staging of diseases, evaluation of therapy effectiveness, and prediction of treatment toxicity for cancer and other diseases. Although semiquantitative indices have been used previously for estimates of organ perfusion, ${ }^{7,8}$ quantitative estimates of perfusion are usually based on the application of compartmental models, with outputs obtained via the solving of a set of nonlinear equations in a multidimensional space. Modeling and measurement of perfusion in the liver present additional complexities due to its dual sources of blood flow, both in terms of data fitting and duration of data acquisition. ${ }^{6,9-11}$ Furthermore, it is desirable to be able to map the distribution of perfusion values throughout the liver. This requires repeated computations on a voxelby-voxel basis.

Thus, calculation of the point-by-point "distribution" of liver perfusion is a computationally daunting task, often precluding the use of these potentially useful volumetric data in clinical trials. In this report, we describe a linear approach to the model fitting with the goal of increasing the efficiency of the data analysis. Simulated data and an example case of liver perfusion of a patient with intrahepatic cancer obtained from DCE CT scans are presented to illustrate the method.

\section{METHOD AND MATERIALS}

Hepatic perfusion is generally estimated using a single compartmental model with dual input functions for the hepatic artery and portal vein using DCE PET, CT, or MRI data. ${ }^{6,-11}$ The liver's dual sources of blood flow also result in a long and dispersed contrast uptake curve in liver parenchyma (e.g., Fig. 1), in which a bolus of intravenously in- jected contrast arrives at the hepatic artery first and $30-40 \mathrm{sec}$ later at the portal vein and liver parenchyma. In order to adequately cover the rapid onset of the first pass of the arterial bolus, and the broad peak, and the slow decay of the portal vein bolus, DCE acquisition has to last at least 90-100 s after an intravenous injection of contrast bolus. Fitting DCE imaging data to a single compartmental model for the liver inherently involves the solving of a nonlinear equation to estimate five free parameters: three representing transfer constants (or blood flow) of contrast from plasma to liver parenchyma or from liver tissue back to central vein, and two denoting time delays of contrast arrival from the hepatic artery and portal vein to liver parenchyma ${ }^{6,9-11}$ (see below).

\section{Liver perfusion model}

Using pharmacokinetics, a change in the contrast concentration of liver parenchyma is given by the differential equation as follows:

$$
\frac{d C_{l}(t)}{d t}=\frac{1}{(1-\mathrm{Hct})}\left[k_{a} C_{a}\left(t-\tau_{a}\right)+k_{p} C_{p}\left(t-\tau_{p}\right)\right]-k_{2} C_{l}(t)
$$

where $C_{l}, C_{a}$, and $C_{p}$ are concentrations of the contrast agent in the respective liver parenchyma, artery, and portal vein, $k_{a}$ and $k_{p}$ are the transfer constants of the contrast agent from respective arterial and portal venous plasma to liver parenchyma, $k_{2}$ is the transfer constant of the contrast agent that leaves the liver parenchyma back to the central vein, $\tau_{a}$ and $\tau_{p}$ are respective delay times of the arrival of artery bolus and portal vein bolus to the liver parenchyma, and Hct is the small vessel hematocrit. A fractional volume of plasma in blood (1-Hct) is considered in Eq. (1) due to the fact that the CT or MRI contrast agent is mostly extra-cellular (does not enter red blood cells) while the concentrations of contrast 


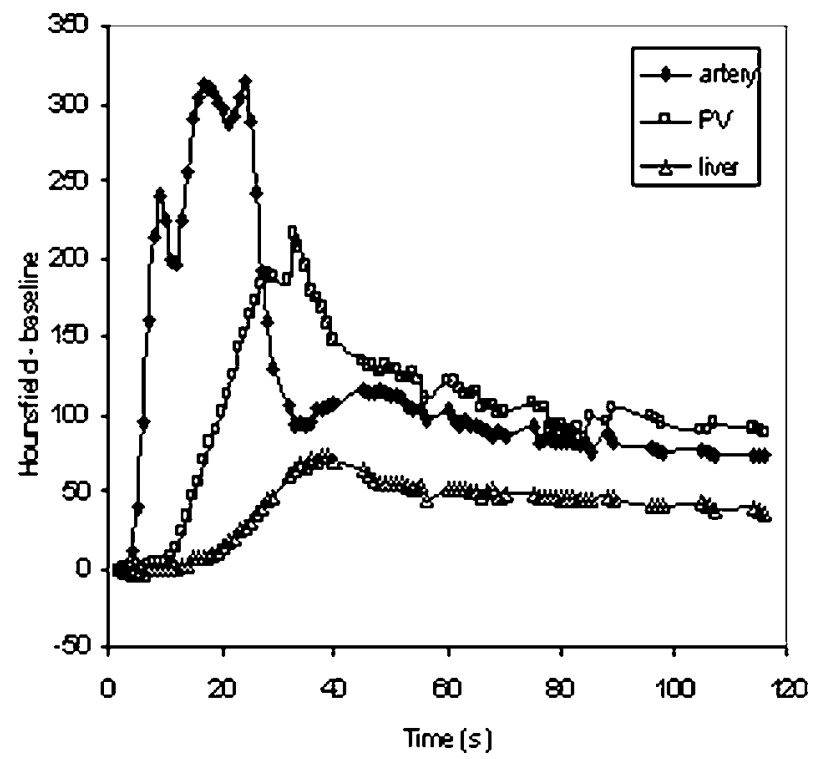

FIG. 1. Time courses of the contrast bolus arrival to aorta (diamond), portal vein (square), and liver parenchyma (triangle) after image registration and data sorting. The peaks of the bolus arrival to portal vein and liver parenchyma delay 30-40 s compared to the onset of the bolus arrival to aorta. The peaks of contrast concentration-time curves in portal vein and liver parenchyma are broad. There are several $\sim 3 \mathrm{~s}$ gaps in the contrast concentrationtime curves for free breathing.

agents in artery and portal vein are determined from blood. Total hepatic blood flow, $F$, is a sum of arterial phase and portal vein phase blood flows,

$$
F=\left(k_{a}+k_{p}\right) / E,
$$

where $E$ is the extraction rate and approximately equal to 1 , which implies the fast exchange limit. In Eq. (1), arterial phase blood flow and portal vein phase blood flow are allowed to vary independently.

\section{Numerical computation}

The solution of the differential equation (1) is given by

$$
\begin{aligned}
C_{l}(t)= & \frac{1}{(1-\mathrm{Hct})} \int_{0}^{t}\left[k_{a} C_{a}\left(\tau-\tau_{a}\right)+k_{p} C_{p}(\tau\right. \\
& \left.\left.-\tau_{p}\right)\right] e^{-k_{2}(t-\tau)} d \tau .
\end{aligned}
$$

A nonlinear least-squares fit (NLLS) of Eq. (3) to DCE data such as those in Fig. 1 can result in unbiased estimates of $k_{a}$, $k_{p}$, and $k_{2}$. However, estimating liver perfusion voxel-byvoxel via NLLS in a five-dimensional parameter space $\left(k_{a}\right.$, $k_{p}, k_{2}, \tau_{a}$, and $\left.\tau_{p}\right)$ is time consuming. Typically, NLLS fitting of Eq. (3) to DCE data is used to estimate $k_{a}, k_{p}$, and $k_{2}$ by minimizing a mean-squared error and using a simplex optimizer $^{12}$ (as was also done here for comparison to the new method below).

Solving a linear equation would be much more desirable and rapid. We first notice that in Eq. (1) the derivative of the contrast concentration-time function of the liver is linearly related to $k_{a}, k_{p}$, and $k_{2}$ as unknown variables with the contrast concentration-time functions of artery, portal vein, and liver parenchyma as known variables. Thus, $k_{a}, k_{p}$, and $k_{2}$ could be obtained by solving the linear equation (1), which would involve calculation of the derivative of the contrast concentration-time function of the liver. However, the error in the computation of the derivative from discrete data is proportional to $\Delta t$ (time interval of temporal sampling), and the noise in data is also amplified in the derivative. We therefore also notice that alternatively, if the integral of Eq. (1) is taken, the linear relationship is still maintained, and we obtain

$$
\begin{aligned}
(1-\mathrm{Hct}) C_{l}(t)= & k_{a} \int_{0}^{t} C_{a}\left(\tau-\tau_{a}\right) d \tau+k_{p} \int_{0}^{t} C_{p}\left(\tau-\tau_{p}\right) d \tau \\
& -(1-\mathrm{Hct}) k_{2} \int_{0}^{t} C_{l}(\tau) d \tau
\end{aligned}
$$

in which the contrast concentration of liver parenchyma is linearly related to $k_{a}, k_{p}$, and $k_{2}$. In Eq. (4), the error in the integral computation from the discrete data is now proportional to $(\Delta t)^{2}$, instead of $\Delta t$ as seen in the derivative approach. Furthermore, the noise in the integral is reduced due to the averaging effect of the time integration of the random noise in each of the three contrast concentration-time functions.

Using matrix notation, a linear least-squares (LLS) solution of Eq. (4) can be obtained for given $\tau_{a}$ and $\tau_{p}$ as

$$
K=\left(A^{T} A\right)^{-1} A^{T} Y,
$$

where

$$
\begin{aligned}
K & =\left(\begin{array}{l}
k_{a} \\
k_{p} \\
-(1-\mathrm{Hct}) k_{2}
\end{array}\right) \text { is a three-dimensional vector, } \\
Y & =\left(\begin{array}{l}
(1-\mathrm{Hct}) C_{l}\left(t_{1}\right) \\
\cdots \\
(1-\mathrm{Hct}) C_{l}\left(t_{i}\right) \\
\cdots \\
(1-\mathrm{Hct}) C_{l}\left(t_{N}\right)
\end{array}\right) \text { is an } N \text {-dimensional vector, }
\end{aligned}
$$

and

$$
A=\left[\begin{array}{l}
\int_{0}^{t_{1}} C_{a}\left(\tau-\tau_{a}\right) d \tau, \int_{0}^{t_{1}} C_{p}\left(\tau-\tau_{p}\right) d \tau, \int_{0}^{t_{1}} C_{l}(\tau) d \tau \\
\cdots \\
\int_{0}^{t_{i}} C_{a}\left(\tau-\tau_{a}\right) d \tau, \int_{0}^{t_{i}} C_{p}\left(\tau-\tau_{p}\right) d \tau, \int_{0}^{t_{i}} C_{l}(\tau) d \tau \\
\ldots \\
\int_{0}^{t_{N}} C_{a}\left(\tau-\tau_{a}\right) d \tau, \int_{0}^{t_{N}} C_{p}\left(\tau-\tau_{p}\right) d \tau, \int_{0}^{t_{N}} C_{l}(\tau) d \tau
\end{array}\right]
$$

is an $N \times 3$ matrix,

with $N$ equal to the number of points in the concentrationtime curves. To compute Eq. (5), first, the $3 \times 3$ symmetric matrix $\left(A^{T} A\right)$ in Eq. (5) is decomposed to $U D U^{-1}$ using sin- 

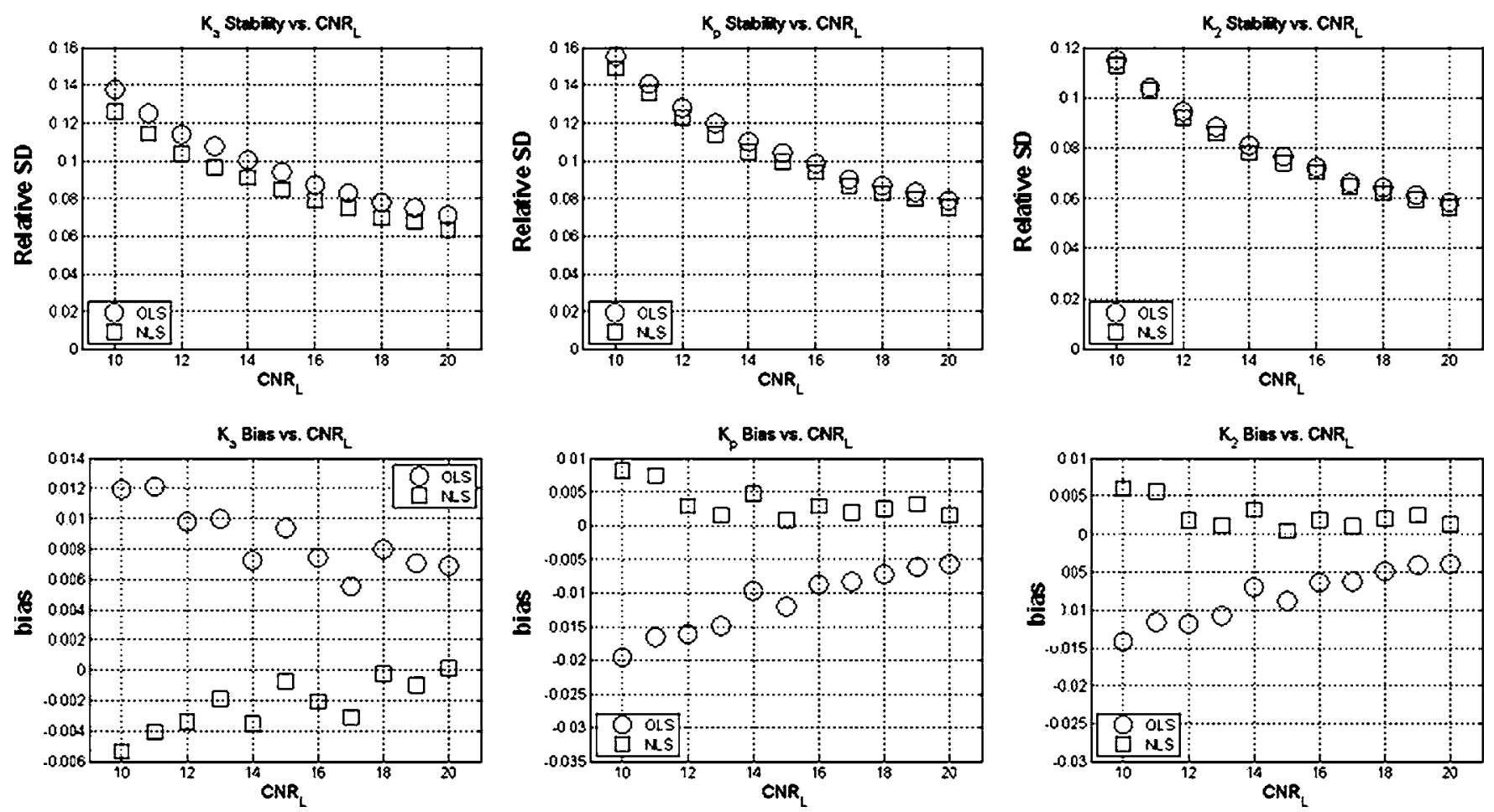

FIG. 2. Scatterplots of relative standard deviations and relative differences vs the contrast-to-noise ratio over 5000 simulations for the three perfusion parameters of $k_{a}, k_{p}$, and $k_{2}$.

gular value decomposition, ${ }^{12}$ where $D$ is a $3 \times 3$ diagonal matrix, $U$ is a unitary matrix, and $U^{-1}$ is its inverse. Then, $K$ is obtained by the matrix product $U D^{-1} U^{-1} A^{T} Y$. Given that delay times of $\tau_{a}$ and $\tau_{p}$ are generally less than $10 \mathrm{~s}$, the computation of each $K$ is repeated while $\tau_{a}$ and $\tau_{p}$ in Eq. (5) are incrementally increased, independently from 0 to $10 \mathrm{~s}$ with a step size of $1 \mathrm{~s}$. The final solution of Eq. (4) is obtained for the $k_{a}, k_{p}, k_{2}, \tau_{a}$, and $\tau_{p}$ that minimize the meansquared errors of Eq. (4). This procedure is performed voxelby-voxel to obtain perfusion maps. Practically, the search ranges for $\tau_{a}$ and $\tau_{p}$ can be chosen and adjusted by evaluation of the fitted results from several voxels or regions of interest. Based upon our experience in DCE CT acquired from ten patients with intrahepatic cancer prior to, during, and after radiation therapy, both delay times are typically $0-2 \mathrm{~s}$, and can be up to $6 \mathrm{~s}$ (further discussions are given below).

All computations were done on a LINUX server (HP Proliant DL740, CPU Intel Xeon $3 \mathrm{GHz}$ ) with an in-house program in $\mathrm{C}++$.

\section{Simulation}

Efficiency, stability, and bias of NLLS and LLS for estimates of perfusion parameters were evaluate by simulated data. The theoretic $C_{a}(t)$ and $C_{p}(t)$ were obtained from the DCE CT data of a patient with intrahepatic cancer (after some data smoothing). The $k_{a}, k_{p}$, and $k_{2}$ values were chosen to be 20,100 , and $450 \mathrm{ml} / 100 \mathrm{~g} / \mathrm{min}$, respectively, which are typical for normal hepatic perfusion. $C_{L}(t)$ was computed from Eq. (3), with $\tau_{a}$ and $\tau_{p}$ set to be initially zero. The typical contrast-to-noise ratio (CNR) values (defined as the ratio of the peak contrast concentration to the standard deviation of baseline signals) were estimated from DCE CT data in normal liver parenchyma of patients with intrahepatic cancer to be 150, 100, and 20 for the aorta, portal vein, and liver parenchyma, respectively (further detailed information is provided in the next section). Thus, Gaussian-distributed random noise was added to the theoretic functions of $C_{a}(t)$ and $C_{p}(t)$ to obtain CNRs of 150 and 100, respectively. Similarly, the CNR for $C_{L}(t)$ was varied between 10 and 20 . The temporal sampling interval, $\Delta t$, of the DCE data was $1 \mathrm{~s}$. For each tested CNR, the perfusion parameters were estimated 5000 times by each of the two methods. For each parameter, the relative standard deviation and the relative difference between the mean of the estimated value and the known value were calculated. The instability of each method was determined by the relative standard deviation of the estimate, and the bias was measured by the relative difference of the mean from the known value. Figure 2 shows that stability for both LLS and NLLS increases with CNR, and differences in stability between the two methods are less than $1.5 \%$ for all three parameters. The bias for both LLS and NLLS is smaller than $2 \%$. The discrepancy between the two methods is tolerable. However, the computation speed is improved by up to 100 times for LLS, compared to NLLS.

Next, a condition representing abnormal perfusion was simulated. The $k_{a}, k_{p}$, and $k_{2}$ values were chosen to be 30 , 50 , and $400 \mathrm{ml} / 100 \mathrm{~g} / \mathrm{min}$, respectively, which replicated the parameters found in the liver parenchyma after it received radiation. Then, the initial $\tau_{a}$ and $\tau_{p}$ values were var- 

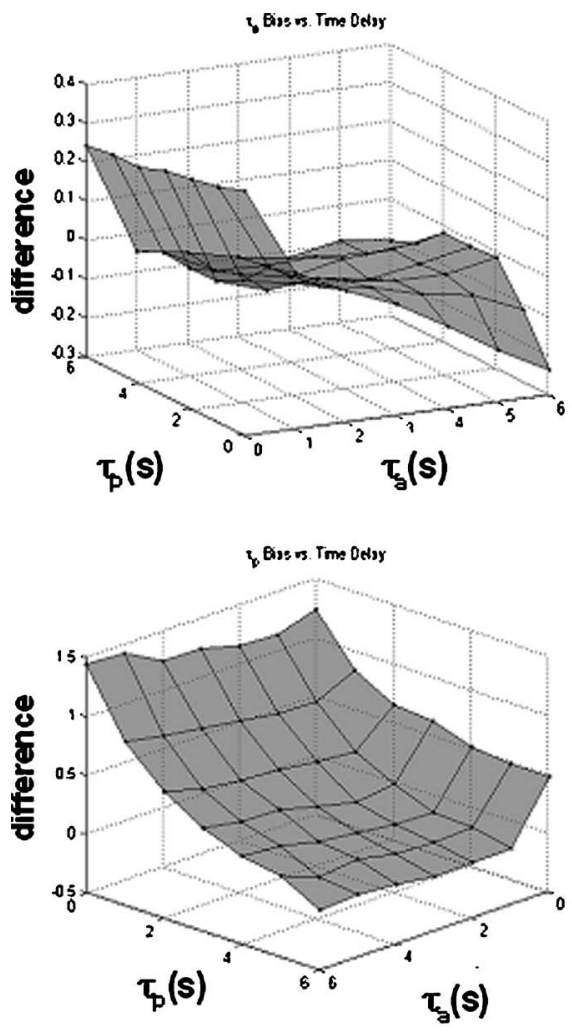
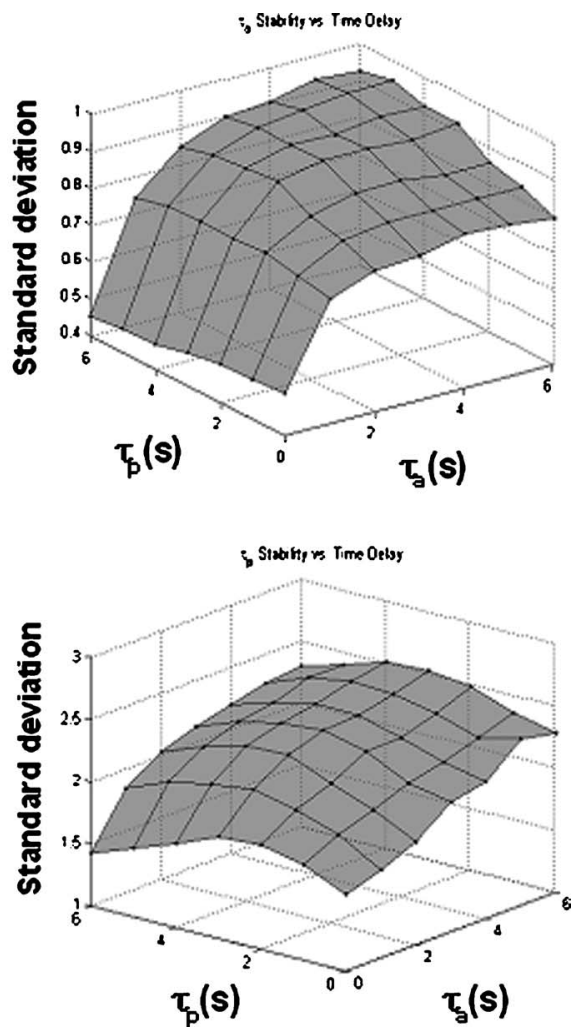

FIG. 3. Three-dimensional scatterplots of differences and standard deviations of estimated $\tau_{a}$ and $\tau_{p}$ over 5000 simulations. ied from 0 to $6 \mathrm{~s}$. Again, Gaussian-distributed random noise was added to $C_{L}(t), C_{a}(t)$, and $C_{p}(t)$ to have a CNR of 20, 150 , and 100, respectively. The searching ranges for $\tau_{a}$ and $\tau_{p}$ were set to be $0-10 \mathrm{~s}$. Again, for each condition, the parameters were estimated 5000 times by LLS. Figure 3 shows that for $\tau_{a}$, differences between means and true values are less than $0.25 \mathrm{~s}$ and standard deviations are less than $1 \mathrm{~s}$ for all conditions. For $\tau_{p}$, differences between mean and true values are less than $1.5 \mathrm{~s}$; the largest difference occurs at $\tau_{p}=0$, and the difference decreases rapidly with an increase in $\tau_{p}$ while standard deviations are less than $2.5 \mathrm{~s}$.

\section{Example case with intrahepatic cancer}

To generate data to demonstrate this fitting procedure (under guidance from an internally reviewed and approved protocol and under informed consent) a patient with intrahepatic cancer was scanned. ${ }^{13}$ The DCE CT scan was performed on a 16-detector multislice scanner (Lightspeed 16, General Electric, Milwaukee, WI), with $2 \mathrm{~cm}$ aperture, $120 \mathrm{KvP}$, $100 \mathrm{~mA}, 1$ rotation per second, and 8 images reconstructed per rotation $(2.5 \mathrm{~mm}$ slice thickness). Prior to the DCE scan, a baseline unenhanced series of $5 \mathrm{~s}$ duration was acquired at controlled exhale using the cine imaging parameters mentioned above. During DCE acquisition, multiple breath hold intervals were used to limit liver position changes and to cover the time course of contrast uptake in the liver up to approximately $120 \mathrm{~s}$, as described in detail elsewhere. ${ }^{13}$ During the first 90-s imaging acquisition, the image volumes were acquired every second. Subsequently, scanning was repeated in three $9 \mathrm{~s}$ intervals with $6 \mathrm{~s}$ gaps for free breathing and $3 \mathrm{~s}$ acquisition for breath hold. The DCE scan resulted in an estimated CT dose index volume $\left(\mathrm{CTDI}_{\mathrm{vol}}\right)$ of $887 \mathrm{mGy}$. We reduced the matrix size of the DCE CT images to 256 $\times 256 \times 8$ from $512 \times 512 \times 8$ by averaging signal intensities of every four pixels in the axial planes. The contrast concentration of the liver described in Eq. (4) is proportional to the difference in enhanced CT signal intensities during contrast uptake and baseline signal intensities in Hounsfield units. The contrast concentrations in artery and portal vein phases were determined from CT voxels that represent aorta and portal vein, respectively. After image registration and data sorting, ${ }^{13}$ we obtained the DCE CT data curves such as those in Fig. 1, in which a time course lasts $120 \mathrm{~s}$ and covers the contrast uptake in artery, portal vein, and liver parenchyma, and has multiple $\sim 3$ s gaps for free breathing.

Figure 4 shows fitting results obtained from NLLS and LLS. The relative difference of residuals of the two fittings was $0.8 \%$. Relative differences of parameters between two fittings were $4 \%, 7.7 \%$, and $7.8 \%$ for $k_{a}, k_{p}$, and $k_{2}$, respectively. However, LLS fitting improved the computation speed by approximately 400 times compared to NLLS fitting. Typically, it takes $\sim 30 \mathrm{~min}$ for computing liver perfusion in a single slice by LLS fitting and $\sim 200 \mathrm{~h}$ by NLLS fitting on a LINUX server of HP Proliant DL740.

Using voxel-by-voxel fitting, we obtained liver perfusion $\left(F=F_{a}+F_{v}\right)$ maps, which are color coded to denote the value of perfusion in units of $\mathrm{ml} / 100 \mathrm{~g} / \mathrm{min}$ and spatially overlaid on the baseline CT images (Fig. 5). Similarly, artery perfusion and portal vein perfusion are displayed separately in Fig. 5. In normal liver parenchyma, typical total perfusion, arterial perfusion, and portal vein perfusion were 128.4, 18.8, and $109.5 \mathrm{ml} / 100 \mathrm{~g} / \mathrm{min}$, respectively. The mean transit 


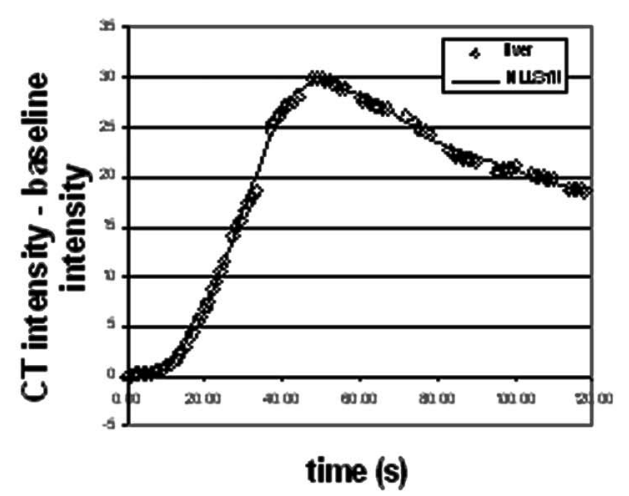

time was $12.7 \mathrm{~s}$. The delay time was $1 \mathrm{~s}$ for both $\tau_{a}$ and $\tau_{p}$. In the center of the tumor (depicted by region of interest 1 in Fig. 5) where the portal vein perfusion is almost zero, $F_{a}=16.3 \mathrm{ml} / 100 \mathrm{~g} / \mathrm{min}, \quad F_{p}=2.4 \mathrm{ml} / 100 \mathrm{~g} / \mathrm{min}, \quad$ MTT $=73 \mathrm{~s}, \tau_{a}=0 \mathrm{~s}$, and $\tau_{p}=6 \mathrm{~s}$. In another part of the tumor (region of interest 2), $F_{a}=44.1 \mathrm{ml} / 100 \mathrm{~g} / \mathrm{min}, \quad F_{p}$ $=15.8 \mathrm{ml} / 100 \mathrm{~g} / \mathrm{min}, \mathrm{MTT}=30.9 \mathrm{~s}, \tau_{a}=1 \mathrm{~s}$, and $\tau_{p}=3 \mathrm{~s}$.

\section{SUMMARY}

A linear least-squares method for estimates of voxel-byvoxel liver perfusion using DCE CT data and a single compartmental and a dual input model has been described here. This method converts a nonlinear problem to a linear problem and reduces the computational time by approximately 100 times compared to nonlinear least-squares fitting, and the differences of fitted parameters between the two methods are acceptable, as demonstrated by simulated data and the DCE CT data from a patient with intrahepatic cancer.

In the single compartmental model, the fast exchange of the contrast agent between the sinusoid and the space of Disse is assumed, which is based upon the fact that in the normal liver the endothelium contains fenestrate of 50-200 nm and has no basement membrane. ${ }^{6}$ For the small molecule size of a contrast agent such as Ultravist 370 ( $700 \mathrm{Da}$ in size) or Gd-DTPA ( 470 Da in size), it is reasonable to assume $E \sim 1$ in Eq. (2). In an abnormal condition, if the exchange rate between the sinusoid and the space of

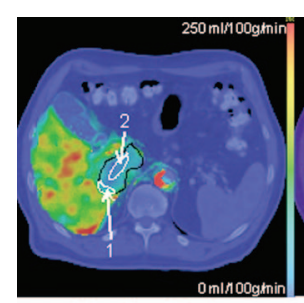

Total perfusion

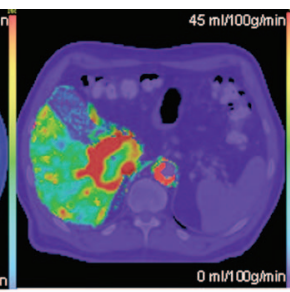

Arterial perfusion

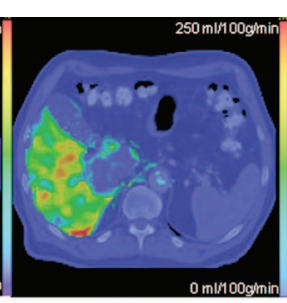

Portal vein perfusion
Fig. 5. Total liver perfusion (left), arterial perfusion (middle), and portal vein perfusion (right) maps (color encoded) obtained by linear least-squares fittings overlaid on a CT image. Perfusion is color coded and denoted in $\mathrm{ml} / 100 \mathrm{~g} / \mathrm{min}$. The black contour depicts the cancer in which the arterial perfusion increases and the portal vein perfusion decreases. Two white contours denote two regions of interest in the tumor.
Disse is reduced but the exchange time (1/rate) is still much shorter than the mean transit time $(1 /$ rate $\ll$ MTT), the single compartment may be still a reasonable assumption. Otherwise, different models need to be considered. ${ }^{10}$

The DCE CT data that were used to test the liver perfusion algorithm came from an IRB-approved clinical study, in which the patients underwent focal hepatic radiation therapy. For this treatment, significant volumes of their livers received radiation doses of 40 Gy or higher. The radiation dose received by this imaging protocol, which was limited to a 2 -cm slab of the liver, was not significant compared to therapeutic dose given to the patients. However, the high dose of the DCE CT may not be adequate for the purpose of diagnosis of patients who are not expected to undergo radiation therapy to the liver. MRI may be an alternative modality to measure liver perfusion. A CNR of 17 was estimated by using a 3D gradient echo pulse sequence with TR of $5.2 \mathrm{~ms}$, TE of $1.8 \mathrm{~ms}$, flip angle of $30^{\circ}$, and voxel size $3 \times 3$ $\times 3 \mathrm{~mm}^{3}$ at 1.5 Tesla. Using state-of-art MRI technologies - 3 Tesla and parallel imaging technologiesfast temporal sampling and high signal-to-noise ratio can be achieved and are more desirable for estimations of liver perfusion parameters.

In summary, this linear least-squares fitting makes voxelby-voxel quantitative estimates of liver perfusion using DCE imaging data and a compartmental model practical for use in clinical trials. Using these perfusion maps, perfusion changes during and after therapy can be assessed for treatment response and for normal tissue toxicity.

\section{ACKNOWLEDGMENTS}

This work was supported in part by NIH Grant Nos. R01 CA85684 and 2 PO1 CA59827.

${ }^{1}$ W. J. Powers and A. R. Zazulia, "The use of positron emission tomography in cerebrovascular disease," Neuroimaging Clin. N. Am. 13, 741758 (2003)

${ }^{2}$ D. G. Nabavi, A. Cenic, R. A. Craen, A. W. Gelb, J. D. Bennett, R. Kozak, and T. Y. Lee, "CT assessment of cerebral perfusion: experimental validation and initial clinical experience," Radiology 213, 141-149 (1999).

${ }^{3}$ R. Hermans, P. Lambin, W. Van den Bogaert, K. Haustermans, A. Van der Goten, and A. L. Baert, "Non-invasive tumour perfusion measurement by dynamic CT: preliminary results," Radiother. Oncol. 44, 159-162 (1997). ${ }^{4}$ R. Hermans, M. Meijerink, W. Van den Bogaert, A. Rijnders, C. Weltens, 
and P. Lambin, "Tumor perfusion rate determined noninvasively by dynamic computed tomography predicts outcome in head-and-neck cancer after radiotherapy," Int. J. Radiat. Oncol., Biol., Phys. 57, 1351-1356 (2003).

${ }^{5}$ L. Ostergaard, A. G. Sorensen, K. K. Kwong, R. M. Weisskoff, C. Gyldensted, and B. R. Rosen, "High resolution measurement of cerebral blood flow using intravascular tracer bolus passages. Part II: Experimental comparison and preliminary results," Magn. Reson. Med. 36, 726736 (1996).

${ }^{6}$ R. Materne, A. M. Smith, F. Peeters, J. P. Dehoux, A. Keyeux, Y. Horsmans, and B. E. Van Beers, "Assessment of hepatic perfusion parameters with dynamic MRI,” Magn. Reson. Med. 47, 135-142 (2002).

${ }^{7}$ T. R. Bader, A. M. Herneth, W. Blaicher, R. Steininger, F. Muhlbacher, G. Lechner, and F. Grabenwoger, "Hepatic perfusion after liver transplantation: noninvasive measurement with dynamic single-section CT," Radiology 209, 129-134 (1998).

${ }^{8}$ M. J. Blomley, R. Coulden, P. Dawson, M. Kormano, P. Donlan, C. Bufkin, and M. J. Lipton, "Liver perfusion studied with ultrafast CT," J. Comput. Assist. Tomogr. 19, 424-433 (1995).
${ }^{9}$ H. Taniguchi, A. Oguro, K. Takeuchi, K. Miyata, T. Takahashi, T. Inaba, and H. Nakahashi, "Difference in regional hepatic blood flow in liver segments-non-invasive measurement of regional hepatic arterial and portal blood flow in human by positron emission tomography with H2(15)O," Ann. Nucl. Med. 7, 141-145 (1993).

${ }^{10}$ H. Taniguchi, A. Oguro, H. Koyama, M. Masuyama, and T. Takahashi, "Analysis of models for quantification of arterial and portal blood flow in the human liver using PET," J. Comput. Assist. Tomogr. 20, 135-144 (1996).

${ }^{11}$ B. E. Van Beers, I. Leconte, R. Materne, A. M. Smith, J. Jamart, and Y. Horsmans, "Hepatic perfusion parameters in chronic liver disease: dynamic CT measurements correlated with disease severity," AJR, Am. J. Roentgenol. 176, 667-673 (2001).

${ }^{12}$ W. H. Press, S. A. Teukolsky, W. T. Vetterling, and B. P. Flannery, Numerical Recipes in C (Cambridge University Press, New York, 1988).

${ }^{13}$ J. M. Balter, J. F. Platt, I. R. Francis, T. S. Lawrence, E. Ben-Josef, Z. Shen, and Y. Cao, "A methodology for CT-based perfusion measurements in the liver," PMB (submitted). 